\title{
Revista Colombiana de

\section{Impacto del aislamiento preventivo obligatorio en la actividad física diaria y en el peso de los niños durante la pandemia por SARS-CoV-2}

\author{
Harold Arévalo ${ }^{\mathrm{a}}$, Miguel Urina Triana ${ }^{\mathrm{b}}$ y Juan Carlos Santacruz ${ }^{\mathrm{c}, *}$ \\ a Médico del deporte, Presidente Asociación De Medicina del deporte de Colombia \\ ${ }^{\mathrm{b}}$ Facultad de Ciencias de la Salud, Universidad Simón Bolívar, Barranquilla, Colombia \\ c Director Ejecutivo Fundación Colombiana del Corazón
}

Recibido el 10 de julio de 2020; aceptado el 4 de septiembre de 2020

Disponible en Internet el 29 de septiembre de 2020

\author{
PALABRAS CLAVE \\ COVID-19; \\ Niños; \\ Aislamiento; \\ Alimentación; \\ Actividad física; \\ Sobrepeso
}

\begin{abstract}
Resumen
Introducción: En Colombia, desde el 24 de marzo hasta el 31 de agosto de 2020, los niños y adolescentes estuvieron 162 días en total aislamiento preventivo obligatorio por la pandemia por COVID-19.

Objetivo: Conocer y evaluar el comportamiento de niños y adolescente durante el período de confinamiento por Covid-19, para establecer las consecuencias sobre la actividad física y el aumento peso.

Métodos: Se elaboró una encuesta virtual abierta realizada en la plataforma de encuestas de Google, dirigida a los padres, la cual estuvo conformada por 5 preguntas: 1. ¿Qué edad tiene su hijo? 2. ¿Cuántos minutos por día de actividad física realiza en casa? 3. ¿Cuántos minutos de pantallas (TV, computador, celular, videojuegos, etc.) ve su hijo al día? 4 ¿Cuántas horas duerme su hijo en la noche? 5. ¿Se ha elevado el peso corporal de su hijo(a)? ¿Cuánto? La encuesta se distribuyó en las bases de datos de la Asociación de Medicina del Deporte y de la Fundación Colombiana del Corazón a través de redes sociales, como WhatsApp, y correos electrónicos. Resultados: Se realizaron 1.139 encuestas virtuales. Se encontró que durante el período de aislamiento obligatorio el $75,2 \%$ de los niños no realizó los minutos recomendados de actividad física por la Organización Mundial de la salud (OMS), que el 82,8\% superó el tiempo de 60 minutos frente a dispositivos electrónicos y que el $44 \%$ aumentó de peso.

Conclusiones: En los períodos de confinamiento es importante diseñar estrategias y procesos educativos que promuevan en los niños estilos de vida que puedan conservar a futuro, cuando sean adultos.

(c) 2020 Sociedad Colombiana de Cardiología y Cirugía Cardiovascular. Publicado por Elsevier España, S.L.U. Este es un artículo Open Access bajo la licencia CC BY-NC-ND (http:// creativecommons.org/licenses/by-nc-nd/4.0/).
\end{abstract}

\footnotetext{
* Autor para correspondencia.

Correo electrónico: jcsantacruz@corazonesresponsables.org (J.C. Santacruz).
} 


\section{KEYWORDS}

COVID-19;

Children;

Isolation;

Feeding;

Physical activity;

Overweight
Impact of mandatory preventive isolation on daily physical activity and weight of children during the SARS-cov-2 pandemic

\begin{abstract}
Introduction: In Colombia from March 24 to August 31, 2020, children and adolescents spent 162 days in total mandatory preventive isolation due to the Covid-19 pandemic.

Objective: To know and evaluate their behavior of children and adolescents during this period of confinement in order to establish the consequences on the physical activity and weight gain. Methods: An open virtual survey was developed on the Google survey platform, addressed to parents with 5 questions: 1 . How old is your child: 2 . How many minutes of physical activity at home do you do? 3. How many minutes of screens (TV, computer, cell phone, video games, etc.) her or she watch per day? 4. How many hours does he or she sleep at night? 5 . Has your child's body weight increased? How much? This survey was distributed in the databases of the Association of Sports Medicine and the Colombian Heart Foundation through social networks such as WhatsApp and emails.

Results: 1,139 virtual surveys were conducted. It was found that during the mandatory isolation period: $75.2 \%$ of the children did not perform the minutes of physical activity recommended by the World Health Organization (WHO), that $82.8 \%$ exceeded the time of 60 minutes versus to electronic devices and that $44 \%$ weight gained.

Conclusions: In periods of confinement, it is important to design educational strategies and processes that promote healthy lifestyles in children that can be preserved in the future in adults.

(C) 2020 Sociedad Colombiana de Cardiología y Cirugía Cardiovascular. Published by Elsevier España, S.L.U. This is an open access article under the CC BY-NC-ND license (http:// creativecommons.org/licenses/by-nc-nd/4.0/).
\end{abstract}

\section{Introducción}

La primera secuencia genérica, identificada por el Instituto Nacional de Salud, estableció que el virus, SARS-CoV-2 llegó a Colombia el 26 de febrero de 2020 y pertenece a la segunda generación de una cadena de transmisión desde un caso importado originado en España ${ }^{1}$.

Un mes después de la detección de primer caso de contagio por COVID-19 en Colombia el gobierno nacional decretó el primer aislamiento preventivo obligatorio, el cual tuvo ocho prórrogas. La cuarentena en todo el país inició el martes 24 de marzo a la media noche y, con la última extensión, finalizó el 31 de agosto ${ }^{2}$. Quiere decir que en total se sumaron 162 días de aislamiento obligatorio para la gran mayoría de colombianos, entre ellos los niños y los adultos mayores de 70 años $^{3}$.

El Aislamiento Preventivo Obligatorio es el período designado para que la ciudadanía permanezca aislada. La medida gubernamental limita totalmente la libre circulación de personas y vehículos en el territorio nacional con excepciones que se fueron aumentando paulatinamente en los decretos que el Gobierno fue dando a conocer con cada extensión o prórroga.

Estas excepciones, especialmente desde la primera semana de mayo, fueron permitiendo la flexibilización del aislamiento ${ }^{4}$.

Para los niños, la primera flexibilización se dio en la primera semana de mayo, lo que indica que los niños permanecieron en cuarentena absoluta 48 días.

Según el Decreto del Gobierno "con el fin de proteger a los niños, niñas y adolescentes de posibles trastornos mentales como depresión o ansiedad, que se pueden presentar por el Aislamiento Preventivo Obligatorio', dispuso que a partir del 11 de mayo los menores entre 6 y 17 años podrían salir de sus casas, por media hora y tres días a la semana, para tomar aire, disfrutar del sol y caminar.

El 23 de junio, cuando el aislamiento obligatorio, se extendió hasta el 15 de julio y después hasta el 31 de agosto, la medida de mayo fue extendida a los niños de 2 a 5 años, y se autorizó que pudieran realizar actividades al aire libre tres veces a la semana durante 30 minutos, alargando a una hora el tiempo concedido a los niños entre 6 y 17 años.

Las medidas hicieron énfasis en la prohibición del uso de patines, bicicletas y balones, así como la utilización de elementos deportivos y juegos de los parques.

La decisión que "busca que haya un momento de esparcimiento de los niños, niñas y adolescentes" delegó en los padres y cuidadores la responsabilidad del cumplimiento de las normas de seguridad sanitaria por parte de los menores, precisando que los niños entre los 2 y 13 años deben estar acompañados por un adulto de entre 18 y 59 años, que no haga parte de la población en riesgo de contagiarse del virus, como adultos mayores o personas con preexistencias de salud.

Además, las medidas complementarias obligaron a los menores de edad a lavarse las manos al salir, usar el tapabocas, mantener un distanciamiento de dos metros y no alejarse del hogar en un perímetro de un kilómetro. Al regreso al hogar se debe hacer un lavado de las suelas de los zapatos, cambiar de ropa y bañar el cuerpo.

El aislamiento obligatorio es inédito en el país, nunca lo había afrontado la población colombiana, y específicamente 
ese aislamiento en los niños empezó a provocar diversas polémicas sobre si era necesario y si fue bien argumentado desde el punto de vista técnico. La evidencia disponible aún es muy poca para sacar conclusiones, en especial porque los estudios del impacto del aislamiento sobre los niños deben evaluar consideraciones sociales, económicas e incluso epidemiológicas.

Los niños son más sintomáticos y transmisores de las enfermedades respiratorias comunes, al punto que es una de las causas más significativas del aumento de la "tasa de mortalidad infantil" en menores de 5 años, según cifras de la Organización Mundial de la Salud ${ }^{5}$. Esta realidad histórica sin embargo no se cumple con el COVID-19, pues los reportes en todos los países que han sufrido el contagio precisan que los niños tienen una tendencia a manifestar de forma más leve la infección por el nuevo coronavirus y que la letalidad es muy baja ${ }^{6}$. Estudios iniciales realizados en China, Estados Unidos, Corea e Italia concluyeron que los niños contagiados en más de un $90 \%$ resultaron asintomáticos o con sintomatología de leve a moderada. Como producto de estos estudios el Imperial College concluyó que la probabilidad de morir por COVID-19, en niños reportados con la enfermedad, en los casos fue de apenas 0,00260\% (Case Fatality Ratio -CFR-) ${ }^{7,8}$.

Por tanto, la decisión de confinar a los niños no se dio pensando en su cuidado, sino en la capacidad que podrían tener de contagiar a otras personas. Por esa razón se cerraron escuelas, colegios y universidades.

Tras las veinte semanas que cumplió el aislamiento obligatorio, al finalizar el 31 de agosto, ha venido surgiendo una fuerte polémica sobre el impacto que esta situación obligada tendrá en la salud física y mental de los niños, por las restricciones impuestas en la práctica de la actividad física, la ausencia de contacto con la naturaleza y la imposibilidad de realizar juegos al aire libre.

De la misma forma ha venido creciendo la polémica sobre el papel de los niños como transmisores del COVID-19. No hay estudios concluyentes que hayan permitido establecer la probabilidad de transmisión de un niño enfermo asintomático o con manifestaciones leves. Tampoco existen estudios de poblaciones que hayan identificado de manera concluyente cuáles son los grupos de edad más propensos al contagio y cuáles son más susceptibles de transmitir la enfermedad. La evidencia más clara permite establecer que el grupo más vulnerable son los adultos mayores que padecen de prexistencias como diabetes, hipertensión, dislipidemias e incluso sobrepeso y obesidad. Los datos disponibles hasta el momento han abierto el debate sobre si los niños son en realidad "peligrosamente transmisores" como se afirmó en un principio".

El gobierno anunció para el mes de septiembre la reapertura de escuelas, colegios y universidades con estrictas medidas sanitarias de seguridad. Entonces se abre una nueva polémica sobre si fue conveniente el cierre y su real impacto sobre la mitigación del contagio por el COVID-19 y sobre la conveniencia de la apertura con las numerosas restricciones y con una comunidad de padres que ha estado sometida a un inusitado cúmulo de información contradictoria, en la mayoría de los casos, que ha exacerbado los comportamientos de temor, miedo y pánico ante la eventual posibilidad de un niño contagiado en el entorno educativo ${ }^{10,11}$.
Adicionalmente muchos padres de familia se debaten en la realidad social de tener que volver en algún momento a su entorno laboral y no encontrar la manera de dejar a sus hijos bajo un cuidado seguro. Muchos niños son hijos de padres que no pueden teletrabajar y que obligatoriamente deberán reincorporarse a sus escenarios de trabajo y otros tantos adolecen de recursos tecnológicos para estudiar en las modalidades virtuales.

Es una realidad difícil de abordar sobre la cual se cierne un escenario de incertidumbre, razón por la cual desde la Asociación de Medicina del Deporte y la Fundación Colombiana del Corazón se quiso contribuir con los resultados de esta encuesta a generar insumos que permitan explorar alternativas que hagan posible reducir el impacto del aislamiento obligatorio en los niños de Colombia.

\section{Metodología}

Para evaluar el impacto del confinamiento en la actividad física diaria y el peso de los niños en Colombia durante el confinamiento se diseñó un instrumento tipo encuesta.

Primero se formularon algunos interrogantes para definir los objetivos. Estas fueron las preguntas formuladas y las respuestas iniciales:

\section{- ¿Qué esperamos aprender de esta encuesta?}

R: Tener un conocimiento sobre el comportamiento de los niños durante el período de confinamiento obligatorio decidido por el Gobierno.

\section{- ¿A quiénes queremos entrevistar?}

R: A los padres de familia que de manera libre decidan contestar la encuesta.

- ¿De qué manera nos ayudará la información obtenida para aportar al debate sobre la conveniencia de aislar a los niños y adolescentes por el peligro de contagio por COVID$19 ?$

R: Los resultados de la encuesta permitirán generar un muestreo sobre el comportamiento de los niños durante la cuarentena para aportar datos sujetos a un análisis objetivo.

Tras definir los objetivos, se diseñó un formulario de encuesta dirigido a padres de familia, el cual fue dispuesto en la plataforma de encuestas de Google, donde se cargó un formulario receptivo, fácil de diligenciar y susceptible de responder a través de cualquier medio digital computador (PC), tableta o celular. La encuesta se distribuyó en las bases de datos de la Asociación de Medicina del Deporte y la Fundación Colombiana del Corazón, utilizando para su difusión las redes sociales como WhatsApp y correo electrónico.

El formulario fue diseñado con la metodología de la encuesta de comportamiento, en la cual se solicitó a los padres de familia responder preguntas acerca de los comportamientos de los niños durante el período de aislamiento preventivo obligatorio generado por la amenaza del contagio por COVID-19 12 . 
Las encuestas de comportamiento se enfocan en lo que las personas hacen y las preguntas se diseñan con respuestas de selección. Son preguntas precisas para que los padres puedan informar sobre el comportamiento de sus hijos. Consecuentemente, las respuestas se consideran un autorreporte de la realidad que observan e interpretan los padres de sus hijos. Esto significa que los resultados obtenidos parten de una consideración subjetiva de acciones individuales, lo cual no disminuye su valor, pues se trata de una realidad que emana de las encuestas de comportamiento y que se tuvo en cuenta en el análisis de los datos.

Se diseñó un cuestionario de 5 preguntas con 5 opciones de respuesta, el cual se define como un cuestionario de datos continuos, tras confirmar que las preguntas seleccionadas recogen la información de interés pretendida en función de los objetivos de la investigación.

Es de resaltar que esta escala de pocos ítems tiene características que favorecen que la fiabilidad sea alta como la definición simple del rasgo a medir y la diversidad de las edades de los niños y adolescentes evaluados.

Las respuestas a las 5 preguntas formuladas les pidieron a los padres de familia evaluar las actividades de sus hijos. Cada pregunta con 5 opciones de respuesta:

Pregunta No. 1 ¿Qué edad tiene su hijo?

- 1 mes a 12 meses

- 12 meses a 3 años

- años a 7 años

- 8 años a 12 años

- Mayor de 13 años

Pregunta No. 2 ¿Cuántos minutos al día de actividad física realiza en casa?

- 30-60 minutos / día

- 60-90 minutos/ día

- 90-120 minutos/día

- Mayor de 120 minutos/día

- Menos de 30 minutos/día

Pregunta No. 3 ¿Cuántos minutos de pantallas (TV, computador, celular, videojuegos, etc.) al día?

- 0 minutos / día

- 60 minutos/día

- 60-90 minutos/día

- 90-120 minutos/día

- Mayor de 120 minutos/día

Pregunta No. 4 ¿Cuántas horas duerme en la noche?

- 12 a 16 horas/día

- 11 a 12 horas/día

- 10 a 13 horas/día

- 9 a 10 horas/día

- Menos de 8 horas/día

Pregunta No. 5 ¿Se ha elevado el peso corporal de su hijo(a)? ¿Cuánto?
- 0 a 1 kilos

- a 3 kilos

- a 6 kilos

- Más de 7 kilos

- Mantiene el peso

La medición y construcción de los índices y escalas en la tabulación se estructuró codificando el porcentaje de respuestas para cada ítem ofrecido.

La metodología de medición fue la de sumar cada selección y traducirla a porcentajes a partir de considerar el $100 \%$ del total de las respuestas para distribuirlas porcentualmente en cada una de las 5 propuestas.

\section{Tamaño de la muestra}

Dado que la encuesta planteó comprender los comportamientos de la población general, en este caso, y quienes respondieron lo hicieron de manera voluntaria, ellos constituyen la población de muestreo. En Colombia, a partir de datos generados por el DANE, un número de 7 millones de personas tienen hijos entre 1 mes y 17 años, que son el objeto de esta investigación ${ }^{13}$. Para que la muestra tenga un nivel de confianza aceptable decidimos un margen de error inferior al $5 \%$, con lo que la muestra por millón de personas debe aproximarse a 150 formularios, lo cual da un total de 1.050 respuestas mínimas para garantizar la confianza de los resultados.

De esta forma se distribuyeron un número de 10.000 encuestas para alcanzar un índice de respuestas del 10\% que garantizara llegar a completar las 1.050 de la muestra.

En total fueron verificadas 1.139 encuestas.

Este tipo de muestra es válida como estudio piloto, lo cual quiere decir que los resultados y su respectivo análisis no tienen un carácter definitivo y sus limitaciones están especialmente circunscritas a la realidad socioeconómica de la muestra y a la localización geográfica de los encuestados, quienes en una amplia mayoría se encuentran en ciudades capitales.

\section{Resultados}

Se analizaron los resultados pregunta por pregunta para una mejor comprensión.

Pregunta No. 1 ¿Qué edad tiene su hijo?: La mayoría de las respuestas correspondieron a padres que tienen a sus hijos en edades entre 4 y 12 años, pues al sumar las opciones de 4 a 7 y de 8 a 12 entrega como resultado un $56.8 \%$, ocupando el primer lugar de respuestas el rango comprendido entre 8 y 12 años con un $30.7 \%$ (fig. 1).

Pregunta No. 2 ¿Cuántos minutos de actividad física por día realiza en casa?: Todas las recomendaciones concluyen que es imprescindible que los jóvenes en edad escolar (de 6 a 17 años) inviertan 60 minutos o más cada día en actividades físicas de moderadas a vigorosas porque tienen beneficios sustanciales para la salud. Precisan las recomendaciones que deben combinarse actividades aeróbicas con ejercicios de fortalecimiento óseo y muscular ${ }^{15}$.

La encuesta permitió establecer que el 13\% de los padres reportan que sus hijos superaron los 60 minutos recomendados, en tanto que más de un $30 \%$ acepta que no 


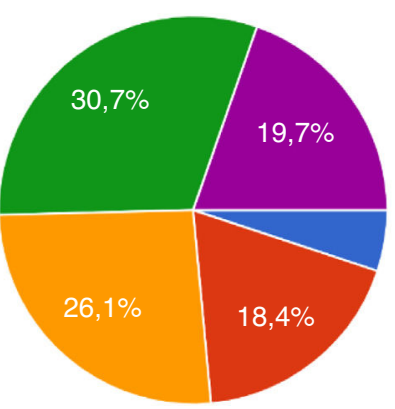

Figura 1
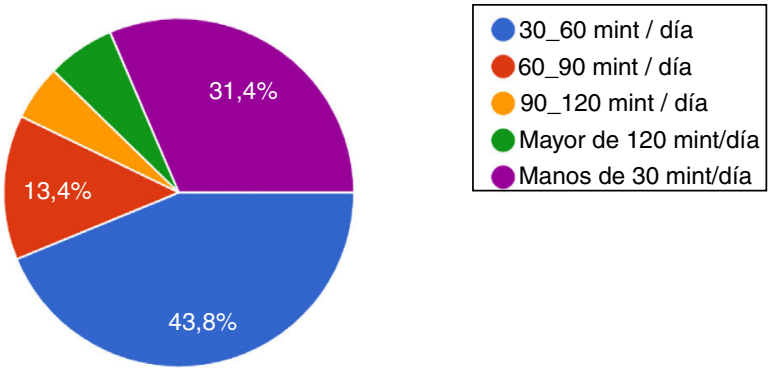

Figura 2

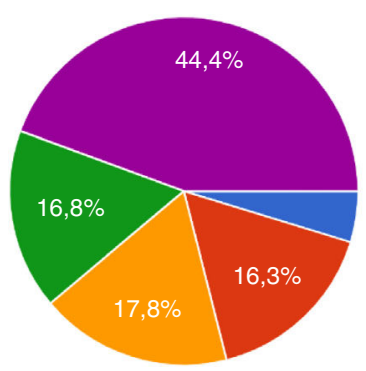

$$
\begin{aligned}
& 0 \text { mint / día } \\
& 60 \mathrm{mint} / \text { día } \\
& 60 \_90 \mathrm{mint} / \text { día } \\
& 90 \_120 \mathrm{mint} / d i ́ a \\
& \text { Mayor de } 120 \mathrm{mint} / \text { día }
\end{aligned}
$$

Figura 3

lograron llegar a 30 minutos, que es la mitad del tiempo recomendado.

Una importante mayoría del 75.2\% afirmó que sus hijos no llegaron al mínimo recomendado, la cual es la cifra más concluyente del análisis de la segunda pregunta del cuestionario (fig. 2).

Pregunta No. 3 ¿Cuántos minutos de pantallas (TV, computador, celular, videojuegos, etc.) ve su hijo por día?: A la ausencia de suficiente tiempo de actividad física, aceptaron los padres de los jóvenes en edad escolar, que durante el período de aislamiento una mayoría absoluta del $82.4 \%$ supera más de 60 minutos frente al televisor, computador, celular o los videojuegos.

Para la Academia Americana de Pediatría los menores de 2 años nunca deberían estar expuestos a pantallas móviles, tabletas, o televisiones, ni siquiera para comer. En esta edad es cuando el cerebro del niño crece más rápidamente, y ellos necesitan el contacto directo con las personas. Con un dispositivo digital el bebé no aprende a hablar, no aprende a comunicarse y tiene un efecto para su capacidad de aprendizaje.

Precisa la Academia Americana de Pediatría, que entre los 2 y los 5 años, el tiempo máximo de dispositivos móvi-

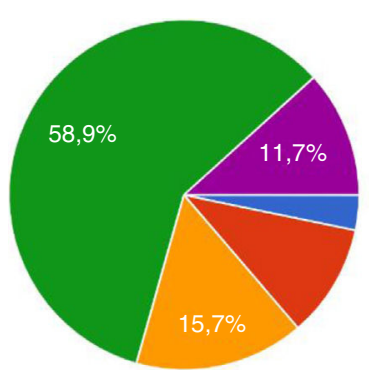

12 a 16 hrs/día

11 a 12 hrs/día

10 a 13 hrs/día

9 a 10 hrs/día

Menos de $8 \mathrm{hrs} / \mathrm{día}$

Figura 4
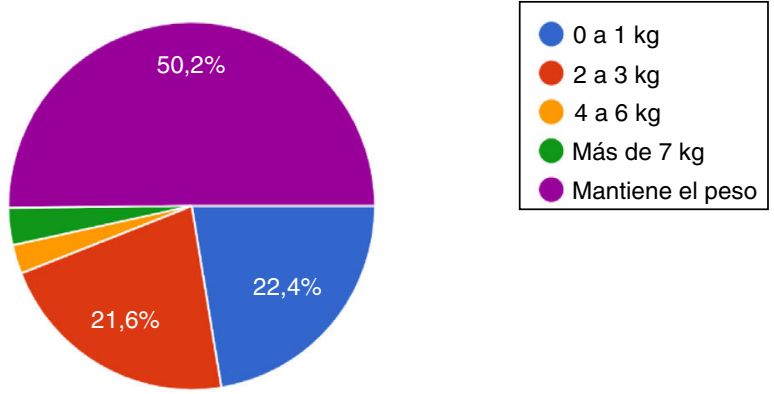

Figura 5

les no debe superar los 30 minutos al día, siempre bajo la supervisión de un adulto para explicar el contenido ${ }^{17}$ (fig. 3).

Pregunta No. 4 ¿Cuántas horas duerme su hijo en la noche?: Los resultados relacionados con las horas de sueño que acumulan los niños, reveló que el $58.9 \%$, quiere decir la mayoría, duermen de 9 a 10 horas, que es el tiempo recomendado de sueño (fig. 4).

Cabe anotar, no obstante, que como se preguntó por las horas de la noche de manera específica, es probable que los padres hayan omitido las siestas.

Las pautas o directrices para el sueño en la infancia según la American Academy of Sleep Medicine, AASM (Academia Estadounidense de la Medicina del Sueño) sostienen que las horas de sueño por día (incluidas siestas o períodos del sueño en el día), deben ser las siguientes:

1. Bebés: 4 a 12 meses. 12 a 16 horas

2. Niños pequeños: 1 a 2 años. 11 a 14 horas

3. Edad preescolar: 3 a 5 años. 10 a 13 horas

4. Edad escolar: 6 a 12 años. 9 a 12 horas

5. Adolescentes: 13 a 18 años. 8 a 10 horas ${ }^{19}$

Pregunta No. 5 ¿Se ha elevado el peso corporal de su hijo(a)? ¿Cuánto?: Si bien más de la mitad de los encuestados, $50.2 \%$, precisó que sus hijos mantuvieron su peso, esta pregunta es, entre las 5 solicitadas, la que entrega menos confiabilidad, pues para determinar la realidad es necesario disponer en el hogar de una báscula para realizar el seguimiento (fig. 4).

No obstante, los padres que aceptaron que sus hijos se subieron entre 1 y 3 kilos durante el período de aislamiento, llegó al 44\%. Se concluye, por tanto, que el $44 \%$ de los niños en edad escolar, especialmente, aumentaron de peso durante los días de confinamiento.

Una combinación de inactividad física con comportamientos sedentarios tiene como consecuencia inevitable el aumento de peso (fig. 5). 


\section{Discusión}

Alrededor del mundo se vienen realizando varias investigaciones para establecer el impacto que las cuarentenas han tenido en los niños y adolescentes; algunas de ellas son Effects of COVID-19 Lockdown on Lifestyle Behaviors in Children with Obesity Living in Verona, Italy: A Longitudinal Study. Obesity, 202020; COVID-19-Related School Closings and Risk of Weight Gain Among Children. Obesity (Silver Spring) 21 y Projecting the impact of the coronavirus disease19 pandemic on childhood obesity in the United States: A microsimulation model. 22

La metodología aplicada con una encuesta de comportamiento es una buena forma de obtener una muestra representativa de una realidad autorreportada, y si bien no se tomaron como ejemplo encuestas previas fundamentadas en evidencia de aplicación y no admite contraste con otras similares, como insumo para el análisis de las realidades que han vivido los niños durante el período del aislamiento obligatorio preventivo es de un inobjetable valor al confirmar, con cifras claras, algunas de las conclusiones que se dan como verdaderas sin que estén avaladas en una medición.

Los resultados obtenidos son producto de una tabulación en porcentajes de las cinco opciones brindadas en cada pregunta.

El cuestionario aplicado tiene limitaciones especialmente en la pregunta 5, pues la respuesta está mediada por la percepción del peso que los padres observan en sus hijos, ya que no se puede tener la seguridad de que realizaron la medida en una báscula para establecer la realidad.

No obstante, cada uno de los datos obtenidos son concluyentes y rotundos, concretamente en el evidente incremento de las actividades sedentarias y el decrecimiento de la actividad física en los niños como consecuencia del confinamiento.

Los resultados y su interpretación se convierten en un insumo destacado para continuar los estudios e investigaciones sobre las consecuencias de las determinaciones gubernamentales ante la llegada del nuevo coronavirus COVID-19 en la población de niños y adolescentes en Colombia.

Es importante señalar respecto a la edad de quienes más respondieron la encuesta, que en estos rangos de edades se encuentran los niños en crecimiento con edad escolar entre 6 y 12 años, justamente la edad que significa un mayor reto para los padres de familia, pues es el momento en que se inicia de lleno la formación en la escuela o colegio de las principales características del relacionamiento con otros niños, el gusto por los juegos y las actividades deportivas, la interacción y la cercanía con sus padres ${ }^{14}$.

Es justamente en estas edades en las que los padres se confrontan sobre los aciertos y desaciertos en la formación y la manera cómo el entorno empieza a impactar de manera muy significativa en los niños como elemento pedagógico complementario de los principios esenciales de formación entregados en el hogar en la primera infancia, ubicada en los rangos de edad de 0 a 5 años.

En la edad escolar, de 6 a 12 años es cuando se forman las principales características de la disciplina, el respeto por la autoridad, la responsabilidad sobre sí mismos y su cuidado y se definen límites, lineamientos y expectativas, pero en especial es cuando se empiezan a manifestar señales claras de una creciente independencia y podrían comenzar los cambios físicos de la pubertad, sobre todo en las niñas.

Son los padres de hijos de este rango de edad quienes están más atentos a participar de este tipo de cuestionarios porque son los que con mayor interés quieren conocer elementos que aporten al proceso formativo en el que se encuentran.

La menor participación es de padres de hijos de 0 a 12 meses, precisamente porque la demanda de los niños en esos momentos está asociada al afecto y al amor con el mayor escenario de dependencia de sus padres y con un impacto mínimo del entorno.

La niñez y la adolescencia son períodos críticos para el desarrollo de habilidades para el movimiento y para aprender conductas saludables orientadas a establecer la base de la salud del futuro adulto, así como la perfección del bienestar asociado a estilos de vida que prioricen los comportamientos orientados al cuidado.

Si la actividad física en niños y adolescentes promueve la salud y el estado físico, este período de cuarentena, superior a los 150 días, dejará en los niños al salir del confinamiento una menor aptitud cardiorrespiratoria y con músculos menos fuertes, entre otras consecuencias.

De la misma forma, y como secuela, puede traducirse en un aprendizaje negativo de comportamientos sedentarios, de acumular más tiempo de ocio improductivo y de perder contacto con la naturaleza y el medio ambiente.

Sumando a lo anterior, es esencial tener en cuenta que la práctica regular de actividad física, que supere los 60 minutos diarios, tiene para los jóvenes en edad escolar beneficios de invaluable impacto para su cerebro, que incluyen mejora cognoscitiva, estímulo de las funciones de la memoria, velocidad de procesamiento, mejor capacidad de atención y mayor rendimiento académico ${ }^{16}$.

Para la edad escolar (6-12 años), quienes mayoritariamente respondieron la encuesta, sostiene la Academia Americana de Pediatría, que ese tiempo no debe superar 60 minutos al día y señala que más tiempo favorece el sedentarismo y la obesidad.

El período de aislamiento puede beneficiar de manera significativa los comportamientos sedentarios gracias a menor actividad física y más tiempo dedicado a los dispositivos móviles. Como consecuencia podríamos tener más jóvenes familiarizados con el control de un joystick que lanzando una pelota.

El"'producto"' del aislamiento puede traer como consecuencia niños más débiles, más lentos y pesados comparados con ellos mismos antes de iniciarse la coyuntura sanitaria y evidenciará lo que se conoce como la tríada de inactividad pediátrica (PIT) con trastorno de déficit de ejercicio, dinapenia pediátrica y analfabetismo físico ${ }^{18}$.

Es importante tener que cuenta que los niños crecen mejor si tienen una rutina nocturna habitual. No acumular las horas de sueño diarias puede desembocar en problemas del comportamiento, salud y control de emociones como irritabilidad, dificultad para concentrarse, hipertensión, 
obesidad, cefalea y depresión. Los niños que duermen bien logran tener un sistema inmunitario más sano, lo cual favorece un mejor rendimiento académico, comportamiento, memoria y salud mental.

\section{Conclusiones}

El confinamiento en niños, sin una estrategia sobre la actividad física y sobre la alimentación balanceada, produce sobrepeso y obesidad

En nuestro estudio el período de aislamiento obligatorio produjo que el $75.2 \%$ de los niños no lograran sumar los minutos recomendados de actividad física, que el $82.8 \%$ superaran el tiempo de 60 minutos recomendados diariamente frente a dispositivos electrónicos y que el $44 \%$ se subiera de peso.

La encuesta tuvo un importante nivel de confianza, exploró la realidad del comportamiento de los niños durante el período de cuarentena que completó en Colombia 162 días y permitió obtener resultados que pueden convertirse en indicadores para el diseño de estrategias orientadas a lograr que los niños puedan tener acceso a mejores oportunidades de formación, conocimiento y educación sobre la importancia de incluir comportamientos de cuidado en su entorno, lo cual implica generar procesos pedagógicos orientadores para padres de familia y profesores, de manera que se logre inducir a los niños desde la infancia en la importancia de incluir conductas saludables en su vida cotidiana.

Se requiere elaborar estrategias educativas que acompañen la realización de actividad física y dietas balanceadas para que en tiempo de aislamiento obligatorio los niños puedan prevenir el aumento de peso.

Desde los entornos educativos se debe priorizar la apropiación de estilos de vida para el cuidado de la salud de manera que se posible sembrar en los niños conductas saludables que puedan mantenerse en su futura edad adulta.

\section{Bibliografía}

1. MSPS. Colombia confirma su primer caso de COVID-19 [Internet]. Ministerio de salud. 2020 [cited 9 July 2020]. Disponible en: https: / /www.minsalud.gov.co/Paginas/Colombia-confirmasu-primer-caso-de-COVID-19.aspx.

2. Presidencia de la República. Decreto 1076 del 28 de julio de 2020, en el cual se ordena la ampliación del Aislamiento Preventivo Obligatorio en todo el país hasta el 31 de agosto. [cited 28 July 2020]. Disponible en: https://id.presidencia.gov.co/Paginas/prensa/2020/GobiernoNacional-expidio-Decreto-1076-que-amplia-AislamientoPreventivo-Obligatorio-hasta-el-30-de-agosto-200729.aspx.

3. Presidencia de la República de Colombia. Decreto 457, mediante el cual se imparten instrucciones para el cumplimiento del Aislamiento Preventivo Obligatorio de 19 días en todo el territorio colombiano. [cited 9 July 2020]. Disponible en: https://id.presidencia.gov.co/Paginas/prensa/2020/GobiernoNacional-expide-Decreto-457-mediante-el-cual-imparteninstrucciones-para-cumplimiento-Aislamiento-Preventiv200323.aspx.

4. Presidencia de la República de Colombia. Junio 2020. Decreto 749, mediante el cual ordena el Aislamiento Preventivo Obligatorio en el país a partir del 1(de junio. [cited 9 July 2020]. Disponible en: https://id.presidencia.gov.co/Paginas/prensa/2020/GobiernoNacional-expide-Decreto-749-mediante-el-cual-ordenaAislamiento-Preventivo-Obligatorio-en-el-pais-a-partir200528.aspx.

5. Reducción de la mortalidad en la niñez. [Internet]. Who.int. 2019. [cited 9 July 2020]. Disponible en: $\quad$ https://www.who.int/es/news-room/factsheets/detail/children-reducing-mortality.

6. Organización Mundial de la Salud. Brote de enfermedad por coronavirus (COVID-19). [Internet]. Febrero 2020. [cited 9 July 2020]. Disponible en: https://www.who.int/es/emergencies/diseases/novelcoronavirus-2019.

7. Campus CS. Coronavirus (COVID-19) - updates and guidance [Internet]. Imperial College London. 2020 [cited 9 July 2020]. Disponible en: https://www.imperial.ac.uk/about/covid-19/.

8. Gallagher L, Campus S. Declines in COVID-19 cases not due to herd immunity, says analysis | Imperial News | Imperial College London [Internet]. Imperial News. 2020 [cited 9 July 2020]. Disponible en: https://www.imperial.ac.uk/news/198238/declines-covid19-cases-herd-immunity-says/.

9. UNICEF. Impacto del COVID-19 en los niños, niñas, adolescentes y sus familias en América Latina y el Caribe. [Internet]. Unicef.org. 2020 [cited 9 July 2020]. Disponible en: https://www.unicef.org/lac/informes/impacto-del-covid19-en-los-ninos-ninas-adolescentes-y-sus-familias-en-alc.

10. Human Rights Watch. Devastador impacto del COVID-19 para niños y niñas | Human Rights Watch [Internet]. Human Rights Watch. 2020 [cited 9 July 2020]. Disponible en: https://www.hrw.org/es/news/2020/04/09/devastadorimpacto-del-covid-19-para-ninos-y-ninas.

11. Centre for Genomic Regulation (CRG). Investigación pediátrica sobre el impacto de COVID-19 en niños. [Internet]. Crg.eu. 2020 [cited 9 July 2020]. Disponible en: $\quad$ https://www.crg.eu/es/content/research/covid-19projects/investigacion-pediatrica-sobre-el-impacto-de-covid19-en-ninos.

12. Knowledge, Attitude and Practice Surveys in Child Protection [Internet]. Resource Centre. 2020 [cited 9 July 2020]. Disponible en: https://resourcecentre. savethechildren.net/node/7245/pdf/kap_report_sp_hires_0.pdf.

13. DANE. ¿Sabías qué? | DANE para Niños, Niñas y Adolescentes. [Internet]. Dane.gov.co. 2020 [cited 9 July 2020]. Disponible en: https://www.dane.gov.co/files/dane-para-ninos/sabiasque.html.

14. American Academy of Pediatrics Escuela primaria [Internet]. HealthyChildren.org. 2020 [cited 9 July 2020]. Disponible en: https://www.healthychildren.org/spanish/agesstages/gradeschool/paginas/default.aspx.

15. Physical Activity Guidelines for Americans. Current Guidelines | health.gov [Internet]. Health.gov. 2020 [cited 9 July 2020]. Disponible en: https://health.gov/our-work/physicalactivity/current-guidelines.

16. Organización Mundial de la Salud. La actividad física en los jóvenes [Internet]. Who.int. 2020 [cited 9 July 2020]. Disponible en: https://www.who.int/dietphysicalactivity/factsheet_youngpeople/es/.

17. American Academy of Pediatrics. Tiempo de dispositivos digitales en niños. [Internet]. Who.int. 2020 [cited 9 July 2020]. Disponible en: https://services.aap. org $/$ en $/$ search $/$ ?context $=$ Healthy\%20Children\&source = Healthy children. org\&lang = Spanish\&k $=$ tiempo\%20de\%20ni\%c3\%b1os $\% 20$ frente $\% 20$ a $\% 20$ dispositivos\%20digitales\&s=. 
18. Faigenbaum AD, Rebullido TR, MacDonald JP. Pediatric Inactivity Triad: A Risky PIT. Curr Sports Med Rep. 2018 Feb;17:45-7, http://dx.doi.org/10.1249/JSR. 0000000000000450. PMID: 29420346.
19. Paruthi S, Brooks LJ, D’Ambrosio C, Hall WA, Kotagal S, Lloyd RM, et al. Recommended amount of sleep for pediatric populations: a consensus statement of the American Academy of Sleep Medicine. J Clin Sleep Med. 2016;12:785-6. 